\title{
The Seek Control Design with Gain-Scheduling in Hard Disk Drives
}

\author{
Eun-Ju Hwang, Chang-Ho Hyun ${ }^{+}$and Mignon Park ${ }^{+}$ \\ School of Electrical and Electronic Engineering, Yonsei University \\ 134 Shinchon-dong, Seodaemun-gu, Seoul, 120-749, Korea
}

\begin{abstract}
The increased disk rotational velocity to improve the data transfer rate has raised up many serious problems in its servo control system which should control the position and velocity of a spot relative to a rotating disk. This paper proposes gain-scheduling-based track-seek control for single stage actuator of hard disk drives. Gain scheduling is a technique that can extend the validity of the linearization approach to a range of operating points and one of the most popular approaches to nonlinear control design. The proposed method schedules controller gains to improve the transient response and minimize overshoot during the functions of the read/write head positioning servomechanism for the seek control. The validity of the proposed method is demonstrated through stability analysis and simulation results.
\end{abstract}

Key Words : Seek control, Gain-scheduling, single-stage actuator, Hard-disk drives.

\section{Introduction}

Hard disk drives (HDDs) have been an important datastorage medium for computers and other data-processing systems. The disk drive industry has developed through dramatic changes in recent decades. The most significant trend in disk drives is that track density and storage capacity are increasing rapidly while access time is being reduced. This trend has led to the need for improved performance of the headpositioning servo system in order to accurately maintain the selected head position along the center of the track and to provide rapid movement of the head from one track to another selected track [1]. Demand for larger capacity, smaller dimensions and higher data transfer rate are some of the driving forces directly linked to the demand for increasing storage density [2]. Decreasing the HDD track width is a feasible idea to achieve these objectives.

Track density is correlative to the track width. Tracks should be written as close as possible to maximize the data-storage on the disk surface. Therefore the track density should be increased, which means a more rigorous requirement of the position of the heads from the true track centre [3].

However, the increased disk rotational velocity to improve the data transfer rate has caused many serious problems. The presence of disturbance in the rotary actuator results in large residual errors and high-frequency oscillations, which may produce larger positioning error signal to hold back the further decreasing of the track width and to deteriorate the performance of the servo systems [4].

One of the main functions of the read/write head positioning servomechanism in HDDs is track seeking. Track seeking

Manuscript received Feb. 10, 2011; revised Feb. 24, 2011;

Accepted Feb. 25, 2011.

${ }^{+}$Corresponding Authors;mignpark@yonsei.ac.kr This research was supported by the research grant of the Kongju National University in 2009 moves the read/write head from the present track to a destination track in minimum time using a control effort. Track seek control is a point-to-point control, and track-to-track maneuver of the heads must be performed in as short as possible. It is difficult to satisfy both the requirements of minimum-time seek using a linear controller. A linear controller can be optimized to meet the specifications of disturbance rejection and good tracking performance. Gains of the controller required to meet these specifications when the position error is low cause actuator saturation when the reference command is several tracks. Performance of linear control deteriorates under control saturation. Although the time optimal control or bang-bang control is a well known solution for point-to-point maneuver, practical realization of the bangbang control has several drawbacks such as control chatter and sensitivity to parameter variations. The basic limitation of the linearization approach is that the controller is guaranteed to operate only in some neighborhood of a single operating point [5], [6]. Therefore, we introduce gain scheduling, a technique that can extend the validity of the linearization approach to a range of operating points. Gain-scheduling is one of the most popular approaches to nonlinear control design and has been widely and successfully applied in many fields. In many situations, it is known how the dynamics of a system change with its operating points. It might be possible to model the system that the operating points are parameterized by one or more variables, which we call scheduling variables [7], [8]. In this case, we may linearize the system at several equilibrium points, design a linear feedback controller at each point, and implement the resulting family of linear controllers as a single controller whose parameters are changed by the scheduling variables. The concept of gain scheduling is intimately connected with the track-to-track maneuver of seek control [9].

Track seek control is a point-to-point control, and track-totrack maneuver of the heads must be performed in as short as possible. Gain scheduling extends the validity of the linearization approach to a range of operating points and 
consists of a family of a linear controller which can be optimized to meet the specifications of disturbance rejection and good tracking performance. Therefore, gain scheduling is one of the most effective technique for seek control for reducing the effects of parameter variations. Furthermore, a good tracking performance and small settling time of servo actuators near a fixed operating point can be achieved by a gain-scheduled controller.

In this paper, a gain scheduling control algorithm for reducing access time and tracking randomly varying parameters in HDDs seek control is proposed. In addition, simulation results are presented to show its efficiency of the proposed controller.

This paper is organized as follows. Section II describes gainscheduling control. In section III, seek control of controlled model is described. Section IV presents simulation results. Finally, some concluding remarks are given in section V.

\section{Gain-Scheduling Control}

In many cases it is possible to find measurable variables that correlate well with changes in process dynamics. These variables can be used to change the controller parameters. This approach is called gain scheduling because the scheme was originally used to measure the gain and then change, that is, schedule, the controller to compensate for changes in the process gain. A good tracking performance and small settling time of servo actuators with controlled position near a fixed operating point can be achieved by a gain-scheduled controller.

Gain scheduling is a powerful technique that is straightforward and easy to use. The key problem is to find suitable scheduling variables, that is, variables that characterize the operating conditions. Typical choices are filtered versions of process input, process output or external variables. It may also be a significant engineering effort to determine the schedules. This effort can be reduced significantly by using automatic tuning because the schedules can then be determined experimentally. Auto-tuning or adaptive algorithms may be used to build gain schedules. A scheduling variable is first determined [10].

The development of a gain-scheduled tracking controller follows these three steps. First, the nonlinear model is linearized about a family of operating points, parameterized by the scheduling variables. Second, using linearization, a parameterized family of linear controllers is designed to achieve the specified performance at each operating point. Finally, a gain-scheduled controller is constructed such that the closed-loop system under the gain-scheduled controller has the same equilibrium point as the closed-loop system under the fixed-gain controller.

Consider the system

$$
\begin{aligned}
& \dot{x}=f(x, u, v, w) \\
& y=h(x, w) \\
& y_{m}=h_{m}(x, w)
\end{aligned}
$$

where $f, h$ and $h_{m}$ are twice continuously differentiable functions in $(x, u, v)$ and continuous in $w$ in a domain $D_{x} \times D_{u} \times D_{v} \times D_{w} \subset R^{n} \times R^{p} \times R^{q} \times R^{l}$. Here, $x$ is the state, $u$ is the control input, $v$ is a measured exogenous input, $w$ is a vector of unknown constant parameters and disturbances, $y \in R^{p}$ is the controlled output, and $y_{m} \in R^{m}$ is measured output.

A gain-scheduled controller can be obtained from the fixedgain controller by scheduling the gains, continuously differentiable functions of $\alpha$, as functions of the scheduling variable $\rho$, that is, replacing $\alpha$ by $\rho$. We represent the gainscheduled controller as

$$
\begin{gathered}
\dot{\varphi}=F(\rho) \varphi+G_{1}(\rho) e+G_{2}(\rho) \dot{y}_{m} \\
\dot{\eta}=L(\rho) \varphi+M_{1}(\rho) e+M_{2}(\rho) \dot{y}_{m} \\
u=\eta+M_{3}(\rho) e
\end{gathered}
$$

When this controller is applied to the nonlinear system, it results in the closed-loop system

$$
\begin{gathered}
\dot{\chi}=g(\chi, \rho, w) \\
y=h(x, w)
\end{gathered}
$$

where

$$
\begin{gathered}
\chi=\left[\begin{array}{l}
x \\
\varphi \\
\eta
\end{array}\right], g(\chi, \varphi, w)=\left[\begin{array}{c}
f\left(x, \eta+M_{3}(\varphi) e, v, w\right) \\
F(\rho) \varphi+G_{1}(\rho) e+G_{2}(\rho) \dot{y}_{m} \\
L(\rho) \varphi+M_{1}(\rho) e+M_{2}(\rho) \dot{y}_{m}
\end{array}\right] \\
e=h(x, w)-r, \dot{y}_{m}=\frac{\partial h_{m}}{\partial x}(x, w) f\left(x, \eta+M_{3}(\varphi) e, v, w\right)
\end{gathered}
$$

When $\rho=\alpha$, the system (4)-(5) has a unique equilibrium point

$$
\chi_{s s}(\alpha, w)=\left[\begin{array}{c}
x_{s s}(\alpha, w) \\
0 \\
u_{s s}(\alpha, w)
\end{array}\right]
$$

at which $y=\alpha_{r}$.

Linearization of (4)-(5) about $\chi=\chi_{\text {ss }}$ and $y=\alpha$ yields

$$
\begin{gathered}
\dot{\chi}_{\delta}=A_{m s}(\alpha, w) \chi_{\delta}+B_{m s}(\alpha, w) \rho_{\delta} \\
y_{\delta}=C_{m s}(\alpha, w) \chi_{\delta}
\end{gathered}
$$

where

$$
\begin{aligned}
& \chi_{\delta}=\chi-\chi_{s s}, \\
& A_{m s}=\left[\begin{array}{ccc}
A+B M_{3} C & 0 & B \\
G_{1} C+G_{2} C_{m}\left(A+B M_{3} C\right) & F & G_{2} C_{m} B \\
M_{1} C+M_{2} C_{m}\left(A+B M_{3} C\right) & L & M_{2} C_{m} B
\end{array}\right] \\
& B_{m s}=\left[\begin{array}{cc}
-B M_{3} C & E \\
-G_{1}-G_{2} C_{m} B M_{3} & G_{2} C_{m} B \\
-M_{1}-M_{2} C_{m} B M_{3} & M_{2} C_{m} B
\end{array}\right] \\
& C_{m s}=\left[\begin{array}{lll}
C & 0 & 0
\end{array}\right]
\end{aligned}
$$




$$
P=\left[\begin{array}{ccc}
I & 0 & 0 \\
G_{2} C_{m} & G_{1} & F \\
M_{2} C_{m} & M_{1} & L
\end{array}\right]
$$

is nonsingular and

$$
P^{-1} A_{m s} P=A_{f}, \quad P^{-1} B_{m s}=B_{f}, \quad C_{m s} P=C_{f}
$$

If measurement of $\dot{y}_{m}$ is not available, we can use the gainscheduled controller

$$
\begin{gathered}
\dot{\varphi}=F(\rho) \varphi+G_{1}(\rho) e+G_{2}(\rho) \vartheta \\
\dot{\eta}=L(\rho) \varphi+M_{1}(\rho) e+M_{2}(\rho) \vartheta \\
u=\eta+M_{3}(\rho) e
\end{gathered}
$$

where $\dot{y}_{m}$ is replaced by its estimate $\vartheta$, provided by the filter

$$
\begin{gathered}
\varepsilon \dot{\zeta}=-\zeta+y_{m} \\
\vartheta=\frac{1}{\varepsilon}\left(-\zeta+y_{m}\right)
\end{gathered}
$$

where $\varepsilon$ is a "sufficiently small" positive constant and the filter is always initiated at $\zeta(0)$ such that

$$
\left\|\zeta(0)-y_{m}(0)\right\| \leq k \varepsilon
$$

for some $k>0$. Since $y_{m}$ is measured, we can always meet this initial condition. Furthermore, whenever the system is initiated from an equilibrium point, the condition (16) is automatically satisfied, since, at equilibrium, $y_{m}=\zeta$. The filter (14)-(15) acts as a derivative approximator when $\varepsilon$ is sufficiently small, as it can be seen from its transfer function

$$
\frac{s}{\varepsilon s+1} I
$$

which approximates the differentiator transfer function $s I$ for frequencies much smaller than $\frac{1}{\varepsilon}$.

The closed-loop system under the gain-scheduled controller (11)-(15) takes the singularly perturbed form

$$
\begin{gathered}
\dot{\chi}=g(\chi, \rho w)+N(\rho)\left(\vartheta-\dot{y}_{m}\right) \\
\varepsilon \dot{\vartheta}=-\vartheta+\dot{y}_{m} \\
y=h(x, w)
\end{gathered}
$$

where

$$
\begin{aligned}
& \dot{y}_{m}=\frac{\partial h_{m}}{\partial x}(x, w) f\left(x \eta+M_{3}(\rho) e v w\right) \\
& \mathrm{N}=\left[\begin{array}{c}
0 \\
G_{2} \\
M_{2}
\end{array}\right]
\end{aligned}
$$

Setting $\varepsilon=0$ results in $\vartheta=\dot{y}_{m}$ and the system (17) - (19) reduces to the system (4) - (5). The next theorem justifies the use of the filter (14) - (15) for sufficiently small $\varepsilon$. Figure 1 shows the realization of the gain-scheduled controller.

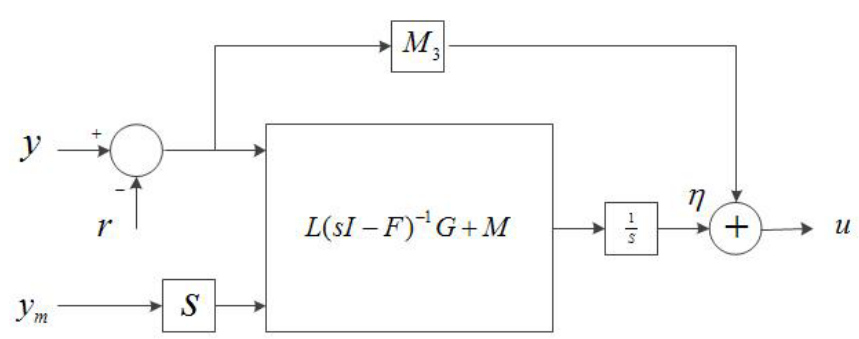

Fig 1. Diagram of Gain-Scheduling controller

\section{Theorem 1}

Consider the closed-loop system (17) - (19) under the stated assumptions. Suppose $\rho(t)$ is continuously differentiable, $\rho(t) \in S \quad$ (a compact subset of $D_{\rho}$, and $\|\dot{\rho}(t)\| \leq \mu$ for all $t \geq 0$. Then, there exist positive constants $k_{1}, k_{2}, k_{3}, k$ and $T$ such that if $\mu<k_{1},\left\|\chi(0)-\chi_{s s}(\rho(0))\right\|<k_{2}$, and $\varepsilon<k_{3}$, then $\chi(t)$ will be uniformly bounded for all $t \geq 0$ and $\|e(t)\| \leq k \mu, \quad \forall t \geq T$.

Furthermore, if $\rho(t) \rightarrow \rho_{\text {ss }}$ and $\dot{\rho}(t) \rightarrow 0$ as $t \rightarrow \infty$, then $e(t) \rightarrow 0$ as $t \rightarrow \infty$.

Proof:

To analyze the closed-loop system (17)-(19), we combine stability analysis of slowly varying system with stability analysis of singularly perturbed systems. We write (17)-(19) as

$$
\begin{gathered}
\dot{\chi}=g(\chi, \rho)+N(\rho)[\vartheta-\phi(\chi, \rho)] \\
\varepsilon \dot{\vartheta}=-\vartheta+\phi(\chi, \rho)
\end{gathered}
$$

When $\varepsilon=0$, we obtain the reduced system $\dot{\chi}=g(\chi, \rho)$ by using the quadratic Lyapunov function $\chi_{\delta}{ }^{T} P_{m s} \chi_{\delta}$. It can be verified that $\phi(\chi, \rho)$ is continuously differentiable in a domain $D_{\chi} \times D_{\rho}$. The change of variables

$$
Y=\chi-\chi_{s s}(\rho), Z=\vartheta-\phi(\chi, \rho)
$$

transforms the system (20)-(21) into

$$
\begin{gathered}
\dot{Y}=g\left(Y+\chi_{s s}(\rho), \rho\right)+N(\rho) \mathrm{Z}-\frac{\partial \chi_{s s}}{\partial \rho} \dot{\rho} \\
\dot{\varepsilon} \dot{Z}=-Z-\varepsilon \frac{\partial \phi}{\partial \chi}\left[g\left(Y+\chi_{s s}(\rho), \rho\right)+N(\rho) \mathrm{Z}\right]-\frac{\partial \chi_{s s}}{\partial \rho} \dot{\rho}
\end{gathered}
$$

Using $v=Y^{T} P_{m s} Y+(1 / 2) Z^{T} Z$ as a Lyapunov function for (22)-(23), we obtain

$$
\begin{aligned}
& \dot{v}=-Y^{T} Y+2 Y^{T} P_{m s}\left[g\left(Y+\chi_{s s}(\rho), \rho\right)-A_{m s}(\rho) Y\right. \\
& \left.+N(\rho) Z-\frac{\partial \chi_{s s}}{\partial \rho} \dot{\rho}\right]+Y^{T}\left[\frac{d}{d t} P_{m s}(\rho)\right] Y \\
& -\frac{1}{\varepsilon} Z^{T} Z-Z^{T}\left\{\frac{\partial \phi}{\partial \chi}\left[g\left(Y+\chi_{s s}(\rho), \rho\right)+N(\rho) Z\right]+\frac{\partial \phi}{\partial \rho} \dot{\rho}\right\} \\
& \leq-\|Y\|_{2}^{2}-\frac{1}{\varepsilon}\|Z\|_{2}^{2}+c_{1}\|Y\|_{2}^{3}+c_{2}\|Y\|_{2}\|Z\|_{2} \\
& +c_{3}\|Y\|_{2}\|\dot{\rho}\|_{2}+c_{4}\|Y\|_{2}^{2}\|\dot{\rho}\|_{2}+c_{5}\|Z\|_{2}{ }^{2}+c_{6}\|\mathrm{Z}\|_{2}\|\dot{\rho}\|_{2}
\end{aligned}
$$


in some neighborhood of the origin, for some positive constants $c_{i}$. Limiting our analysis to a neighborhood where $\|Y\|_{2} \leq c_{7} \leq 1 /\left(4 c_{1}\right)$ we arrive at the inequality

$$
\begin{aligned}
& \dot{v} \leq-\frac{1}{2}\|Y\|_{2}^{2}-\frac{1}{2 \varepsilon}\|\mathrm{Z}\|_{2}^{2}+\left(c_{3}\|Y\|_{2}\right. \\
& \left.+c_{4} c_{7}\|Y\|_{2}+c_{6}\|\mathrm{Z}\|_{2}\right)\|\dot{\rho}\|_{2} \\
& -\left[\begin{array}{l}
\|Y\|_{2} \\
\|\mathrm{Z}\|_{2}
\end{array}\right]^{T}\left[\begin{array}{cc}
1 / 4 & -c_{2} / 2 \\
-c_{2} / 2 & 1 /(2 \varepsilon)-c_{5}
\end{array}\right]\left[\begin{array}{l}
\|Y\|_{2} \\
\|\mathrm{Z}\|_{2}
\end{array}\right]
\end{aligned}
$$

Choosing $\varepsilon^{*}$ small enough that the $2 \times 2$ matrix is positive definite for all $0<\varepsilon<\varepsilon^{*}$ we end up with

$$
\dot{v} \leq-2 \alpha v+2 \beta \sqrt{v}\|\dot{\rho}\|_{2}
$$

for some positive constants $\alpha$ and $\beta$. Hence, $W=\sqrt{v}$ satisfies the inequality

$$
D^{+} W \leq-\alpha W+\beta\|\dot{\rho}\|_{2}
$$

Applying the comparison lemma concludes the proof [8].

This theorem shows that if the scheduling variable is slowly varying and the initial state is sufficiently close to the equilibrium point at the initial time, then the tracking error will tend to zero if the scheduling variable approaches a constant limit.

\section{Seek Controller Design}

Consider the second-order system

$$
\begin{aligned}
& \dot{x}_{1}=\tan x_{1}+x_{2} \\
& \dot{x}_{2}=x_{1}+u \\
& y=x_{2}
\end{aligned}
$$

where $y$ is the only measured signal; that is, $y_{m}=y$. We want $y$ to track a reference signal $r$. We use $r$ as the scheduling variable. We want to design an output feedback controller that achieves small tracking error $e=y-r$ in response to the exogenous input

$$
\rho=\left[\begin{array}{l}
r \\
v
\end{array}\right] \in D_{\rho}=D_{r} \times D_{v}
$$

We use integral control to achieve zero steady-state error when $v=\alpha$ and rely on gain scheduling to achieve small error for slowly varying $\rho$. We partition $\alpha$ as $\alpha=\left[\begin{array}{ll}\alpha_{r}{ }^{T}, & \alpha_{v}{ }^{T}\end{array}\right]^{T}, \alpha_{r}$ and $\alpha_{v}$ are constant values for $r$ and $v$, respectively. We use $\rho$ as the scheduling variable. For the design of integral control, we assume that there is a unique pair $\left(x_{s s}, u_{s s}\right): D_{\rho} \times D_{w} \rightarrow D_{x} \times D_{u}$, continuously differentiable in $\alpha$ and continuous in $w$, such that

$$
\begin{gathered}
o=f\left(x_{s s}(\alpha, w), u_{s s}(\alpha, w), \alpha_{v}, w\right) \\
\alpha_{r}=h\left(x_{s s}(\alpha, w), w\right)
\end{gathered}
$$

for all $(\alpha, w): D_{\rho} \times D_{w}$.

When $r=\alpha=$ constant, the equilibrium equation(24) and (25) have the unique solution

$$
x_{s s}(\alpha)=\left[\begin{array}{c}
-\tan ^{-1} \alpha \\
\alpha
\end{array}\right], u_{s s}(\alpha)=\tan ^{-1} \alpha
$$

We use the observer-based integral controller

$$
\begin{aligned}
& \dot{\sigma}=e=y-r \\
& \dot{\hat{x}}=A(\alpha) \hat{x}+B u+H(\alpha)(y-C \hat{x}) \\
& u=-K_{1}(\alpha) \hat{x}-K_{2}(\alpha) \sigma
\end{aligned}
$$

where

$$
\begin{aligned}
& A(\alpha)=\left[\begin{array}{cc}
1+\alpha^{2} & 1 \\
1 & 0
\end{array}\right], B=\left[\begin{array}{l}
0 \\
1
\end{array}\right], C=\left[\begin{array}{ll}
0 & 1
\end{array}\right] \\
& K_{1}(\alpha)=\left[\left(1+\alpha^{2}\right)\left(3+\alpha^{2}\right)+3+\frac{1}{1+\alpha^{2}} \quad 3+\alpha^{2}\right], K_{2}(\alpha)=-\frac{1}{1+\alpha^{2}} \\
& H(\alpha)=\left[\begin{array}{c}
10+\left(4+\alpha^{2}\right)\left(1+\alpha^{2}\right) \\
\left(4+\alpha^{2}\right)
\end{array}\right]
\end{aligned}
$$

Since $\dot{y}$ is not available, we implement the gain-scheduled controller (11)-(15) with $\varepsilon=0.01$.

\section{Simulation Results}

The feedback gains $K_{1}(\alpha)$ and $K_{2}(\alpha)$ are designed to assign the closed-loop eigenvalues at $-1,-(1 / 2) \pm j(\sqrt{3} / 2)$. The observer gain $H(\alpha)$ is designed to assign the observer eigenvalues at $-(2 / 3) \pm j(3 \sqrt{3} / 2)$. We have chosen the eigenvalues independent of $\alpha$ for convenience, but we could have allowed them to depend on $\alpha$ as long as their real parts are less than a negative number independent of $\alpha$.

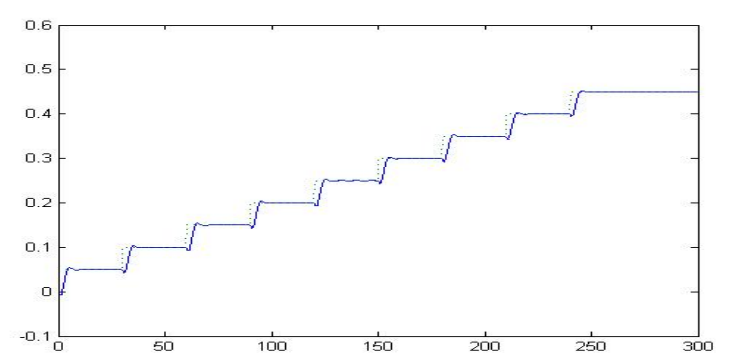

Fig. 2. The reference (dashed) and output (solid) signals of the gain-scheduled controller for the step inputs

Figure 2 shows the response of the closed-loop system to a sequence of step changes in the reference signals. The output signals track to the reference step signals well. 


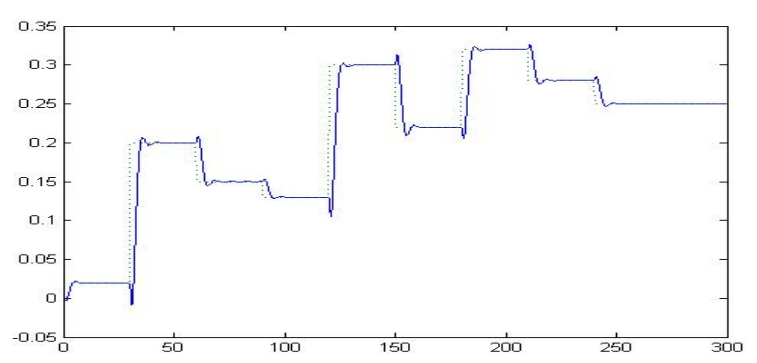

Fig. 3. The reference (dashed) and output (solid) signals of the gain-scheduled controller for the random inputs

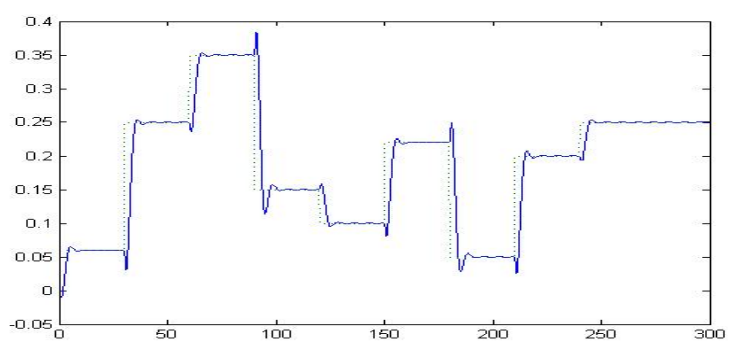

Fig. 4. The reference (dashed) and output (solid) signals of the gain-scheduled controller for the random inputs

Figure 3 and figure 4 shows the response of the closed-loop system to random changes in the reference signals. The output signals track well to the reference signals which are randomly changed.

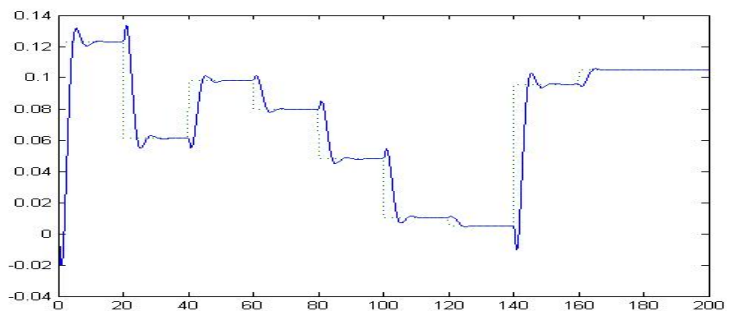

Fig. 5. The reference (dashed) and output (solid) signals of the gain-scheduled controller for the random inputs

Figure 5 represents the response of the closed-loop system to random changes in the reference signals in relatively short time.

The output signals track well to the reference signals which are randomly and slightly changed in a short time. The simulation results represents that the proposed controller satisfies with the demand of accurate and rapid movement of the head from one track to another selected track.

\section{Conclusion}

Gain scheduling based on measurements of operating conditions of the process is often a good way to compensate for variations in process parameters. Gain scheduling is an effective technique that is straightforward and easy to use for seek control for reducing the effects of parameter variations. Furthermore, a good tracking performance and small settling time of servo actuators near a fixed operating point can be achieved by a gain-scheduled controller. In this paper we have focused on seek control of HDDs adapted gain-scheduling controller. When the system parameters are changed for track seeking, gain-scheduled controller makes the tracking error zero and achieves the desired stability and performance requirements. The simulation results show that the proposed algorithm is successfully adapted for reducing access time and tracking randomly varying parameters in HDDs seek control.

\section{References}

[1] B. M. Chen, T. H. Lee and V. Venkataramanan, Hard Disk Drive Servo Systems. Springer, Great Britain, 2002.

[2] M. Kobayashi and R. Horowitz, "Track seek control for hard disk dual-stage servo systems," IEEE Transactions on Magnetics, vol.37, no.2, pp. 949-954, 2001.

[3] A. A. Mamun, G. Guo, C. Bi, Hard Disk Drive. CRC press, Boca Raton, 2007.

[4] J. Ryoo, K. Jin, T. Doh, M. Chung, "New fine seek control for optical disk drives," in Proc. American Control Conference, pp. 3635-3639, 1999.

[5] V. F. Montagner and P. L. D. Peres, "State feedback gain scheduling for linear systems with time-varying parameters," Journal of Dynamic Systems, Measurement, and Control, vol. 128, no. 2, 2006.

[6] B. M. Isayed and M. A. Hawwa, "A nonlinear PID control scheme for hard disk drive servosystems," in Proc. IEEE conf. Control and Automation, pp. 1-6, 2007.

[7] P. A. Ioannou and J. Sun, Robust Adaptive Control. Prentice Hall, New Jersey, 1996.

[8] H. K.Khalil, Nonlinear Systems. Prentice Hall, New Jersey, 2002.

[9] D. J. Leith and W. E. Leithead, "Survey of gain-scheduling analysis and design," International Journal of Control, vol. 73, no. 11, pp. 1001-1025, 2000.

[10] K.J. Åström and B. Wittenmark, Adaptive Control, Dover Publications,Inc., 2008. 


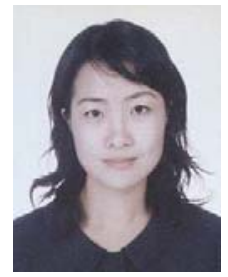

Eunju Hwang received the B.S. degrees in metallurgy engineering and material science from Hongik University, Seoul, Korea, in 1999, and the M.S. degrees in electronic engineering from Yonsei University, Seoul, Korea, in 2006. She is currently a Ph. D. candidate of Dept. of electrical and electronic engineering in Yonsei University.

Her current research interests include intelligent control, robust control, adaptive control, Hard disk drives, and robot manipulate.

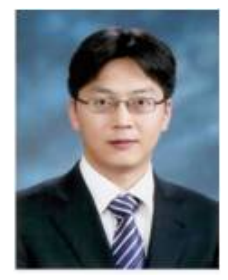

Chang-Ho Hyun received the B.S. degrees in control and instrumentation engineering from Kwangwoon University, Seoul, Korea, , and the M.S. and Ph.D. degrees in electrical and electronic engineering from Yonsei University, Seoul, Korea, in 1999. 2002, 2008. From 2008 to 2009, he was a senior engineering in Samsung Electronics. Since 2009, he has joined the faculties of the School of Electrical, Electronic and Control Engineering at Kongju National University, where he is currently a full-time lecturer. His current research interests include intelligent control and application, nonlinear control, robotics, mobile robots.

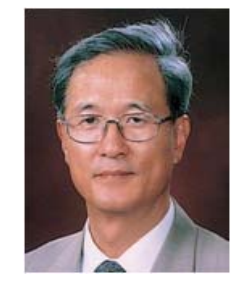

Mignon Park received a B.S. and an M.S. in electronics from Yonsei University, Seoul, Korea, in 1973 and 1977, and a Ph.D. from the University of Tokyo, Tokyo, Japan, in 1982. He was a researcher in the Institute of Biomedical Engineering, University of Tokyo, from 1972 to 1982 , as well as at the Massachusetts Institute of Technology, Cambridge, and the University of California Berkeley, in 1982. $\mathrm{He}$ was a visiting researcher in the Robotics Division, Mechanical Engineering Laboratory Ministry of International Trade and Industry, Tsukuba, Japan, from 1986 to 1987 . He has been a Professor in the Department of Electrical and Electronic Engineering, Yonsei University, since 1982. His research interests include fuzzy control and applications, robotics, and fuzzy biomedical systems.

E-Mail : mignpark@yonsei.ac.kr (M. Park) 